MPP-2013-152

\title{
Dimensional Oxidation of Non-geometric Fluxes in Type II Orientifolds
}

\author{
Ralph Blumenhagen ${ }^{1}$, Xin Gao ${ }^{1,2}$, Daniela Herschmann ${ }^{1}$ and Pramod Shukla ${ }^{1}$ \\ 1 Max-Planck-Institut für Physik (Werner-Heisenberg-Institut), \\ Föhringer Ring 6, 80805 München, Germany \\ 2 State Key Laboratory of Theoretical Physics, \\ Institute of Theoretical Physics, Chinese Academy of Sciences, \\ P.O. Box 2735, Beijing 100190, China
}

\begin{abstract}
Some aspects of string compactifications with non-geometric fluxes are revisited in the light of recent progress in double field theory. After rederiving the general form of these fluxes, we consider the proposed flux induced four-dimensional effective superpotential and oxidize its induced scalar potential to terms in a ten-dimensional action. This analysis is performed independently for an explicit toroidal type IIA and its T-dual type IIB orientifold. We show in detail that the result of this bottom-up approach is compatible with the gauged supergravity motivated flux formulation of the double field theory action in both the NS-NS and the R-R sector.
\end{abstract}




\section{Contents}

1 Introduction

2 Preliminaries 5

2.1 T-duality ........................... 5

2.2 Flux induced superpotential . . . . . . . . . . . . . 6

2.3 Double field theory . . . . . . . . . . . . . . . . 8

2.4 Fluxes and Bianchi identities in DFT . . . . . . . . . . . . . 10

2.5 Coexistence of fluxes for orientifolds . . . . . . . . . . . . . . 13

3 Dimensional oxidation $\quad \mathbf{1 4}$

3.1 A type IIA orientifold . . . . . . . . . . . . . . 14

3.2 Oxidation to $10 \mathrm{D}$ type IIA action . . . . . . . . . . . 16

3.3 A type IIB orientifold . . . . . . . . . . . . . . . . . . 18

3.4 Oxidation to $10 \mathrm{D}$ type IIB action . . . . . . . . . . 21

4 Relation to DFT 22

4.1 Relating the oxidized NS-NS action to DFT . . . . . . . . . 22

4.2 Relating the oxidized R-R action to DFT . . . . . . . . . . . 23

5 Conclusions 24

A The detailed form of the superpotentials 26

B Details on the scalar potential

\section{Introduction}

The application of T-duality to known string solutions with non-trivial three-form flux in connection with the possible gaugings in gauged supergravity has led to the proposal for the existence of non-geometric fluxes in string theory. During the last couple of years, both their string model building implications as well as their mathematical description have been under intense study. This revealed a surprisingly rich structure opening new physical possibilities and connections to current areas of mathematics.

Concretely, successively applying the Buscher rules to a flat three-dimensional background with constant $H$-flux, leads in the first step to a twisted torus with a constant geometric F-flux [1] and in the second step [2, 3, 4], to the nongeometric $Q$-flux. Such backgrounds can be considered to be locally geometric, where the transition functions between two overlapping charts are stringy $\mathrm{T}$ duality transformations. Therefore, such backgrounds have been called T-folds [5]. A final T-duality in the non-isometric third direction was conjectured to lead 
to the $R$-flux [6], for which the background is expected to be not even locally geometric.

Since a Kalb-Ramond three-form supported on a Calabi-Yau threefold was known to generate an effective four-dimensional superpotential, the form of the latter for the non-geometric $Q$ - and $R$-fluxes could be conjectured [6]. Analogously, one could derive that a combination of Ramond-Ramond fluxes and nongeometric fluxes contribute to the R-R tadpole cancellation conditions. In fact, it was argued that these contributions can weaken these constraints in the sense that their effect is like that of anti-branes [7, 8]. Turning on those more general fluxes break the no-scale structure of the simplest type IIB superpotential and the presence of new stable AdS and Minkowski vacua has been shown for toroidal examples. Clearly, it is one of the crucial questions of string phenomenology, whether the flux induced scalar potential (in connection with instanton and Dterm contributions) can stabilize all moduli in a stable or meta-stable de Sitter minimum. Including the non-geometric fluxes this question was approached lately in $[9,10,11,12,13$.

It was pointed out quite early that the stringy fluxes are expected to be closely related to the possible gaugings in gauged four-dimensional supergravity [14, 15, 16, 17, 18, 19, 20, 21]. As a consequence, the induced scalar potentials in four-dimensions should match. Evidence for this was presented in the literature [22, 23, 24, 25] for the concrete case of a $\mathbb{Z}_{2} \times \mathbb{Z}_{2}$ orbifold. In this paper, we will revisit this background with all NS-NS fluxes $H, F, Q, R$ and all R-R fluxes turned on in a type IIA and a type IIB orientifold and analyze it in very much detail, but with special emphasis on their ten-dimensional origin.

It is quite remarkable that this entire four dimensional analysis could be performed without having an honest ten-dimensional framework, which from first principles implements the non-geometric sector of string theory. The various types of fluxes were treated as essentially independent objects, only constrained by a couple of Bianchi identities. Since the number of degrees of freedom of a string is fixed, one would expect that there are additional constraints, which become visible in a just mentioned microscopic description of all the fluxes.

During the last couple of years, the underlying mathematical principles of nongeometry were under intense investigation. Here the main candidates are generalized geometry [26, 27, 28, 29] and double field theory (DFT) [30, 31, 32, 33, 18]. In the former, the $B$-field gauge transformations and the diffeomorphisms are unified in $O(D, D)$ transformations acting on the generalized bundle $T M \oplus T^{*} M$ (see [34, 35, 36] for a generalization to M-theory). In doing so, the theory automatically contains $\beta$-transforms, which, loosely speaking, open the door to (part of) the non-geometric regime. In fact, by performing $O(D, D)$ field redefinitions, tendimensional actions involving the non-geometric $Q$ and $R$-fluxes could be written down [37, 38, 39, 40, 41]. Here, the differential geometry of Lie-algebroids turned out to be the relevant mathematical structure.

However, the detailed analysis of [42] led the authors to the conclusion that 
these redefined actions do not inherently allow to describe non-geometric $Q$ and $R$-flux string backgrounds. The reason behind that is that the new actions only feature the redefined symmetries of the original supergravity action, i.e. diffeomorphisms and $B$-field gauge transformations, but for instance no local $\beta$-transforms. The latter are incorporated into DFT so that this seems to be the more appropriate framework for a global description of non-geometric backgrounds (for pedagogical reviews of DFT please see [43, 44]).

In DFT not only the dimension of the bundle is doubled but even the dimension of the underlying manifold. Motivated by the decoupling of left- and rightmovers on the string world-sheet, besides the ordinary coordinates, one introduces winding coordinates. The latter are the conjugate variables to the winding of the string. In DFT, T-duality acts by exchanging normal with winding coordinates and also allows to perform a T-duality in non-isometric directions. Motivated by string field theory, a $2 D$-dimensional action admitting global $O(D, D)$ invariance as well as invariance under both ordinary diffeomorphisms and winding diffeomorphisms was constructed. Due to the unphysical doubling of the coordinates, this action is supplemented by the strong constraints, which implements the stringy level-matching condition on the level of the effective theory. Thus, one way of thinking about the DFT action is that, in constructing this (leading order) effective string action, one imposes the level-matching condition after computing the string scattering amplitudes.

In this paper, now equipped with the DFT framework, we revisit some aspects of the early approaches to non-geometric fluxes. First of all, generalizing the computation done in [45] to DFT, we rederive the most general form of the $H, F, Q, R$-fluxes in terms of the generalized metric from the closure of the algebra of certain differential operators. The so obtained form is consistent with the earlier results presented in [24, 18, 19]. The Jacobi identities of this Lie-algebra imply the general form of the Bianchi identities, which are also consistent with the recent results reported in [46].

Second and this should be considered as the main objective of this paper, we perform a closer investigation of the T-duality motivated form of the flux induced superpotential [6, 8]. For that purpose, on a simple toroidal orientifold with all invariant geometric and non-geometric NS-NS and R-R fluxes turned on, we present the computation of the induced scalar potential in very much detail. This analysis is carried out for a pair of T-dual type IIA and type IIB orientifolds separately. Note that this computation turns out to be highly nontrivial, as the Bianchi identities of the fluxes have to be invoked many times. Consistent with general expectation, the induced scalar potential is that of a half-maximally gauged supergravity theory.

Such a detailed comparison between the scalar potential induced by the fluxed superpotential and the scalar potential of gauged supergravity was described for a subset of fluxes, namely geometric and $H$-flux, in type IIA in [22]. Beyond that, in [23] is was very briefly stated at the very end of the paper that the authors 
have explicitly verified the matching for all orientifold even fluxes in the type IIB case. Similarly such a result was briefly stated for the special case of an isotropic $T^{6}$ in [25]. 1] Our detailed computation verifies these claims for the type IIB case and provides many interesting details of the general computation in particular for the less studied type IIA case.

Next we oxidize the four-dimensional scalar potential to an underlying tendimensional action. We emphasize that in determining the ten-dimensional origin of the scalar potential, one has to keep in mind that in writing down a superpotential, one is treating the effects of the background fluxes as small perturbations around the Calabi-Yau geometry. As the main result of this paper, we find that, both in the NS-NS and in the R-R sector, the resulting oxidized tendimensional action is compatible with the flux formulation of the DFT action [18, 19, 20, 48, 49]. Therefore, our detailed and explicit computation can be considered to provide another explicit verification of all the four and ten-dimensional concepts developed during the last years to describe non-geometric fluxes.

\section{Preliminaries}

In this section, both for self-consistency and for formulating the problem, we review a couple of relevant issues about non-geometric fluxes. Here we essentially follow the historical development.

\section{$2.1 \quad$ T-duality}

One of the most distinctive features of string theory is certainly T-duality. Applying this transformation to configurations which are already well understood has led to substantial new insights about string theory. Most recently, applying T-duality to closed-string backgrounds with non-vanishing three-form flux, revealed configurations transcending the usual large volume geometric framework. Thus, they have been called non-geometric.

As usual, one defines the NS-NS two-form $B=\frac{1}{2} B_{i j} d x^{i} \wedge d x^{j}$ and its field strength $H=d B=\frac{1}{3 !} H_{i j k} d x^{i} \wedge d x^{j} \wedge d x^{k}$, i.e. $H_{i j k}=3 \partial_{[\underline{i}} B_{j k]}$ 2. Now, one considers a flat three-dimensional background with constant $H$-flux, $H_{123}=h$. Using the gauge symmetry of the $B$-field, one can choose $B_{12}=h x_{3}$. The directions $x_{1}$ and $x_{2}$ are isometries so that one can employ the Buscher rules for a T-duality along these circle directions. As has been shown in detail in [1, 6] a T-duality along $x_{1}$ leads to a twisted three-torus which is characterized by a geometric flux $F_{23}^{1}=h$. A further T-duality along the isometry direction $x_{2}$ leads to a non-standard type of string background, where the transition function

\footnotetext{
${ }^{1}$ Similar to [22, M-theory compactification on $G_{2}$ manifolds with the inclusion of nongeometric F-fluxes have been studied in [4].

${ }^{2}$ The anti-symmetrization of $n$ indices is defined with the inclusion of a prefactor $1 / n$ !.
} 
between two charts is given by a stringy T-duality transformation. This nongeometric twist is characterized by a $Q$-flux, $Q_{3}{ }^{12}=h$. It now seems tempting to also pull the last index up by performing a T-duality in the non-isometry direction $x_{3}$. Clearly, the Buscher rules cannot be applied in this case, but it was speculated that one gets a genuine non-geometric background with constant $R$ flux, $R^{123}=h$. These backgrounds are expected to be not even locally geometric. This chain of transformations can be summarized by

$$
H_{i j k} \stackrel{T_{k}}{\longleftrightarrow} F_{i j}{ }^{k} \stackrel{T_{j}}{\longleftrightarrow} Q_{i}{ }^{j k} \stackrel{T_{i}}{\longleftrightarrow} R^{i j k}
$$

As we will review in section 2.3, a framework to also describe this last Tduality in a non-isometry direction is given by DFT. Here one formally introduces the canonical conjugate variable $\tilde{x}_{i}$ to the winding operator. Thus, the space is doubled and parametrized by standard and winding coordinates $X^{M}=\left(\tilde{x}_{i}, x^{i}\right)$. A T-duality in a certain direction also exchanges the corresponding normal and winding coordinates.

\section{$2.2 \quad$ Flux induced superpotential}

Turning on fluxes on a given string background, like e.g. a torus or a CalabiYau manifold, induces various effects. First, the fluxes contribute non-trivially to the ten-dimensional equations of motion and to the supersymmetry variations. Therefore, generically supersymmetry is broken and the equations of motion are not satisfied any longer. This manifests itself in the four-dimensional effective supergravity theory by an induced scalar potential, which at the level of second order in derivatives arises from a flux induced superpotential or Fayet-Iliopolous term. Just working in the four-dimensional effective theory, the idea is that the potentially new minima of the scalar potential indeed correspond to new true solutions to the ten-dimensional equations of motion, in which the backreaction of the fluxes is taken into account. To show this in detail is however a highly non-trivial issue.

Second, the flux induces not only the just described tadpoles for the dilaton and the graviton, but also for certain Ramond-Ramond p-form gauge fields. Therefore, generically tadpole cancellation requires the introduction of orientifold planes and D-branes in the background.

In this paper we are concerned with the effect of turning on all kinds of geometric and non-geometric fluxes, which can best be seen in type IIA orientifolds. Recall that the string-frame ten-dimensional action for the bosonic fields of the type IIA supergravity is

$$
\begin{aligned}
S_{\text {IIA }}= & \frac{1}{2 \tilde{\kappa}_{10}^{2}} \int d^{10} x \sqrt{-G}\left[e^{-2 \Phi}\left(R+4(\nabla \Phi)^{2}-\frac{1}{2}|H|^{2}\right)-\frac{1}{2}\left|G^{(2)}\right|^{2}-\frac{1}{2}\left|G^{(4)}\right|^{2}\right] \\
& -\frac{1}{4 \tilde{\kappa}_{10}^{2}} \int B \wedge d C^{(3)} \wedge d C^{(3)}
\end{aligned}
$$


with gravitational coupling $\tilde{\kappa}_{10}^{2}=\frac{1}{4 \pi}\left(4 \pi^{2} \alpha^{\prime}\right)^{4}$, which we set to one in the following. Here we used the notation

$$
\left|G^{(p)}\right|^{2}=\frac{1}{p !} G_{M_{1} \ldots M_{p}} G^{M_{1} \ldots M_{p}} .
$$

Note that the field strengths $G^{(p)}$ in the R-R sector also involve the R-R-potentials of lower degree and also the NS-NS two-form $B$ :

$$
G^{(2)}=d C^{(1)}, \quad G^{(4)}=d C^{(3)}-C^{(1)} \wedge H
$$

This leads to correction terms for the usual Bianchi identities, as for instance one gets $d G^{(4)}+H \wedge G^{(2)}=0$. Introducing also a $G^{(0)}$ field strength leads to massive type IIA. The other field strengths receive further corrections

$$
\begin{aligned}
& G^{(2)}=d C^{(1)}-G^{(0)} B, \quad G^{(4)}=d C^{(3)}-B \wedge G^{(2)}-\frac{1}{2} B \wedge B G^{(0)} \\
& G^{(6)}=d C^{(5)}-B \wedge G^{(4)}-\frac{1}{2} B \wedge B \wedge G^{(2)}-\frac{1}{6} B \wedge B \wedge B G^{(0)}
\end{aligned}
$$

and the Bianchi identities can compactly be written as

$$
d\left(e^{B} G\right)=0 \Leftrightarrow d G^{(p)}+H \wedge G^{(p-2)}=0 .
$$

Therefore, it is the combination $e^{B} G$ which is closed in cohomology. We call its background value $\bar{G}$.

In $\mathcal{N}=1$ supersymmetric theories in four dimensions the scalar potential can be written as a sum of two terms, an F-term and a positive semi-definite D-term. The former can be derived from a holomorphic superpotential $W$ and the real Kähler potential $K$ via

$$
V_{W}=e^{K}\left(G^{i \bar{\jmath}} D_{i} W D_{\bar{\jmath}} \bar{W}-3|W|^{2}\right)
$$

Generalizing the celebrated type IIB computation of Taylor/Vafa [50], the superpotential for turning on the R-R fluxes and the H-flux in massive type IIA orientifolds on Calabi-Yau threefolds was derived via dimensional reduction of (2.2) in [51] (see also [52]) and reads

$$
W=\frac{1}{4}\left(-i \int_{X} \bar{H} \wedge \Omega^{C}+\int_{X} e^{i J_{c}} \wedge \bar{G}\right),
$$

where the complexified Kähler modulus is defined as $J_{c}=J+i B$, and the complex structure moduli are encoded in

$$
\Omega^{c}=\operatorname{Re}\left(i e^{-\phi} \Omega_{3}\right)+i C^{(3)} .
$$


Thus, in type IIA the superpotential depends also on the Kähler moduli, but still no terms mixing the complex structure and the Kähler moduli appear.

Applying successive T-duality for a toroidal background, it was argued in [6] (see also [8]) that such mixing terms are generated by the T-dual geometric and non-geometric fluxes. The proposed superpotential reads

$$
W=\frac{1}{4}\left(-i \int_{X} \overline{\mathfrak{H}}^{C} \wedge \Omega^{C}+\int_{X} e^{i J_{c}} \wedge \bar{G}\right),
$$

where the background three-form flux

$$
\overline{\mathfrak{H}}^{C}=\frac{1}{6} \overline{\mathfrak{H}}_{i j k}^{C} d x^{i} \wedge d x^{j} \wedge d x^{k}
$$

is defined as

$$
\begin{aligned}
\overline{\mathfrak{H}}_{i j k}^{C}=\bar{H}_{i j k}+3 \bar{F}_{[\underline{i j}}^{m}\left(-i J_{c}\right)_{m \underline{k}]} & +3 \bar{Q}_{[\underline{i}}^{m n}\left(-i J_{c}\right)_{m \underline{j}}\left(-i J_{c}\right)_{n \underline{k}]} \\
& +\bar{R}^{m n p}\left(-i J_{c}\right)_{m[\underline{i}}\left(-i J_{c}\right)_{n \underline{j}}\left(-i J_{c}\right)_{p \underline{k}]} .
\end{aligned}
$$

As indicated, here the fluxes $\bar{H}, \bar{F}, \bar{Q}$ and $\bar{R}$ receive some background values, whereas $J_{c}$ are still considered as the moduli of the Calabi-Yau threefold. This means that turning on geometric or non-geometric fluxes is treated as a perturbation around the unfluxed Calabi-Yau geometry.

Via the supergravity relation (2.7) the superpotential (2.10) induces a scalar potential involving all closed string moduli. Considering this scalar potential to be generated by the dimensional reduction of a ten-dimensional effective action, it is a natural question to ask how such a ten-dimensional action involving also the non-geometric fluxes must look like. Thus, we want to oxidize the fourdimensional effective action to a ten-dimensional one.

\subsection{Double field theory}

In order to inherently describe non-geometric string backgrounds, where e.g. the transition functions are T-duality transformations, one needs an effective string action which is manifestly invariant under the full $O(D, D)$ group. The search for such an action has led to DFT [33, 30, 31, 32], which so far is only understood at leading order in a derivative expansion.

As already mentioned, the main new feature of DFT is that one doubles the number of coordinates by introducing winding coordinates $\tilde{x}_{i}$ and arranges them into a doubled vector $X^{I}=\left(\tilde{x}_{i}, x^{i}\right)$. One defines an $O(D, D)$ invariant metric

$$
\eta_{I J}=\left(\begin{array}{cc}
0 & \delta^{i}{ }_{j} \\
\delta_{i}{ }^{j} & 0
\end{array}\right)
$$


Moreover, the dynamical fields $G_{a b}$ and $B_{a b}$ are combined in the generalized metric

$$
\mathcal{H}_{I J}=\left(\begin{array}{cc}
G^{i j} & -G^{i k} B_{k j} \\
B_{i k} G^{k j} & G_{i j}-B_{i k} G^{k l} B_{l j}
\end{array}\right) .
$$

Indices are pulled up and down with $\eta$, like for instance

$$
\mathcal{H}^{I J}=\eta^{I I^{\prime}} \mathcal{H}_{I^{\prime} J^{\prime}} \eta^{J^{\prime} J} \text {. }
$$

As in ordinary differential geometry, one can introduce an $O(D, D)$ generalized non-holonomic frame via

$$
\mathcal{H}_{I J}=E^{A}{ }_{I} S_{A B} E^{B}{ }_{J}
$$

where the diagonal matrix $S_{A B}$ is defined as

$$
S_{A B}=\left(\begin{array}{cc}
s^{a b} & 0 \\
0 & s_{a b}
\end{array}\right)
$$

with $s_{a b}$ being the flat $D$-dimensional Minkowski metric. For the parametrization (2.14) of the generalized metric one finds

$$
E_{I}^{A}=\left(\begin{array}{cc}
e_{a}{ }^{i} & -e_{a}{ }^{k} B_{k i} \\
0 & e^{a}{ }_{i}
\end{array}\right)
$$

with $e_{a}{ }^{i} s^{a b} e_{b}^{j}=G^{i j}$.

The full DFT action in $2 D$ dimensions can then be written in terms of the generalized metric as

$$
\begin{aligned}
S_{\mathrm{DFT}} & =\frac{1}{2} \int d^{2 D} X e^{-2 d}\left(\frac{1}{8} \mathcal{H}^{I J}\left(\partial_{I} \mathcal{H}^{K L}\right)\left(\partial_{J} \mathcal{H}_{K L}\right)\right. \\
& \left.-\frac{1}{2} \mathcal{H}^{I J}\left(\partial_{J} \mathcal{H}^{K L}\right)\left(\partial_{L} \mathcal{H}_{I K}\right)-2\left(\partial_{I} d\right)\left(\partial_{J} \mathcal{H}^{I J}\right)+4 \mathcal{H}^{I J}\left(\partial_{I} d\right)\left(\partial_{J} d\right)\right) .
\end{aligned}
$$

Note that here $d^{2 D} X=d^{D} x d^{D} \tilde{x}, \partial_{I}=\left(\tilde{\partial}^{i}, \partial_{i}\right)$, and $d$ denotes the dilaton which is defined as $\exp (-2 d)=\sqrt{-|G|} \exp (-2 \phi)$. This action has been determined by invoking a number of symmetries: First it was required to be invariant under local diffeomorphisms of the coordinates $X^{I}$, i.e. $\left(\tilde{x}_{i}, x^{i}\right) \rightarrow\left(\tilde{x}_{i}+\tilde{\xi}_{i}(X), x^{i}+\xi^{i}(X)\right) 3$. Second, the action is invariant under a global or rigid $O(D, D)$ symmetry, which acts as

$$
\begin{array}{ll}
\mathcal{H}^{\prime}=h^{t} \mathcal{H} h, & d^{\prime}=d, \\
X^{\prime}=h X, & \partial^{\prime}=\left(h^{t}\right)^{-1} \partial
\end{array}
$$

\footnotetext{
${ }^{3}$ The $x^{i}$ dependence of these two diffeomorphisms includes both standard diffeomorphisms and $B$-field gauge transformations. Note that the winding coordinate dependence of $\xi^{i}$ also gives what one might call $\beta$-field gauge transformations.
} 
with

$$
h=\left(\begin{array}{ll}
a & b \\
c & d
\end{array}\right) .
$$

For manifest $O(D, D)$ invariance and for closure of the algebra of infinitesimal diffeomorphisms, this action has to be supplemented by the strong constraint

$$
\partial_{i} A \tilde{\partial}^{i} B+\tilde{\partial}^{i} A \partial_{i} B=0 .
$$

Solving (2.22) via $\tilde{\partial}^{i}=0$, the double field theory action reduces to the familiar action in the geometric frame.

Besides the form of the DFT action (2.19), there exist equivalent ones, which differ by terms that are either total derivatives or are vanishing due to the strong constraint. As will become important later, there exist the so-called flux formulation of the DFT action, which is motivated by the scalar potential in gauged supergravity [18, 19, 20] and which, as shown in [53], is also related to the early work of W.Siegel [54, 55].

One can also parametrize the generalized metric via

$$
\mathcal{H}_{I J}=\left(\begin{array}{cc}
g^{i j}-\beta^{i k} g_{k l} \beta^{l j} & \beta^{i k} g_{k j} \\
-g_{i k} \beta^{k j} & g_{i j}
\end{array}\right)
$$

where $\beta=\frac{1}{2} \beta^{a b} \partial_{a} \wedge \partial_{b}$ denotes an anti-symmetric bi-vector. The geometric frame (2.14) and this non-geometric one are related via the field redefinition

$$
\begin{aligned}
& g=G-B G^{-1} B \\
& \beta=-g^{-1} B G^{-1}
\end{aligned}
$$

which is reminiscent of the Buscher rules. The reduction of the DFT action for this $(g, \beta)$ frame with $\tilde{\partial}^{i}=0$ has been carried out in detail in [38, 39].

\subsection{Fluxes and Bianchi identities in DFT}

The form of the four kinds of fluxes $H, F, Q$ and $R$ in DFT was determined in [24, 18, 19]. Here, we rederive them from the generalization of the Roytenberg algebra from [56, 45] to DFT.

The observation is that the fluxes $H_{a b c}, F^{a}{ }_{b c}, Q_{a}{ }^{b c}$ and $R^{a b c}$ appear as structure "constants" in the Roytenberg algebra [57] on $T X \oplus T^{*} X$

$$
\begin{aligned}
& {\left[e_{a}, e_{b}\right]=F_{a b}^{c} e_{c}+H_{a b c} e^{c},} \\
& {\left[e_{a}, e^{b}\right]=Q_{a}^{b c} e_{c}-F^{b}{ }_{a c} e^{c},} \\
& {\left[e^{a}, e^{b}\right]=R^{a b c} e_{c}+Q_{c}{ }^{a b} e^{c} .}
\end{aligned}
$$

A representation of this algebra can be given by the Lie-bracket of certain vector fields on the tangent bundle of the doubled geometry of DFT. Then, the 
Bianchi identities for these fluxes arise from the Jacobi-identities of this Liealgebra. Generalizing the computation of [45], we consider the following two $B, \beta$-twisted derivative operators 4 .

$$
\mathcal{D}_{a}=\partial_{a}+B_{a m} \tilde{\partial}^{m}, \quad \tilde{\mathcal{D}}^{a}=\tilde{\partial}^{a}+\beta^{a m} \mathcal{D}_{m}
$$

where we have used a non-holonomic basis

$$
\partial_{a}=e_{a}^{i} \partial_{i}, \quad \tilde{\partial}^{a}=e_{i}^{a} \tilde{\partial}^{i}
$$

with $e_{i}^{a} e_{j a}=g_{i j}$ and $e_{a}{ }^{i} e_{i}^{b}=\delta_{a}{ }^{b}$. Here we allow the $e_{a}{ }^{i}$ to depend on both normal and winding coordinates. For the commutator of two partial derivatives one gets

$$
\left[\partial_{a}, \partial_{b}\right]=f_{a b}^{c} \partial_{c}
$$

with

$$
f_{a b}^{c}:=e_{i}^{c}\left(\partial_{a} e_{b}^{i}-\partial_{b} e_{a}^{i}\right)
$$

Analogously, for the partial winding derivatives one finds

$$
\left[\tilde{\partial}^{a}, \tilde{\partial}^{b}\right]=\tilde{f}_{c}^{a b} \tilde{\partial}^{c}
$$

with

$$
\tilde{f}_{a}^{b c}:=e_{a}^{i}\left(\tilde{\partial}^{b} e_{i}^{c}-\tilde{\partial}^{c} e_{i}^{b}\right)
$$

It is now a tedious, though straightforward computation to derive the commutator algebra of the two twisted derivatives (2.27)

$$
\begin{aligned}
& {\left[\mathcal{D}_{a}, \mathcal{D}_{b}\right]=F^{c}{ }_{a b} \mathcal{D}_{c}+H_{a b c} \tilde{\mathcal{D}}^{c},} \\
& {\left[\mathcal{D}_{a}, \tilde{\mathcal{D}}^{b}\right]=Q_{a}{ }^{b c} \mathcal{D}_{c}-F^{b}{ }_{a c} \tilde{\mathcal{D}}^{c},} \\
& {\left[\tilde{\mathcal{D}}^{a}, \tilde{\mathcal{D}}^{b}\right]=R^{a b c} \mathcal{D}_{c}+Q_{c}{ }^{a b} \tilde{\mathcal{D}}^{c}}
\end{aligned}
$$

\footnotetext{
${ }^{4}$ Note that these twisted derivatives are related to the usual ones via a choice of generalized non-holonomic frame

$$
E^{A}{ }_{I}=\left(\begin{array}{cc}
e_{a}{ }^{i} & e_{a}{ }^{k} B_{k i} \\
e^{a}{ }_{k} \beta^{k i} & e^{a}{ }_{i}+e^{a}{ }_{k} \beta^{k l} B_{l i}
\end{array}\right)
$$

which contains both $B$ and $\beta$. There is a change of signs relative to (2.18), but we intend here to use the same convention for the fluxes as in [46]. This slight inconsistency does not affect the computation in section 3 . Note that in (2.27) and therefore also in (2.26) we have made an asymmetric choice in the definition of $\mathcal{D}_{a}$ and $\tilde{\mathcal{D}}^{a}$.
} 
with the following definitions of the $H$-flux

$$
H_{a b c}:=3\left(\partial_{[\underline{a}} B_{\underline{b c]}}+f_{[\underline{a b}}^{m} B_{\underline{c}] m}\right)+3\left(B_{[\underline{a} m} \tilde{\partial}^{m} B_{\underline{b c}]}+B_{[\underline{a} m} B_{\underline{b} n} \tilde{f}_{\underline{c}]}^{m n}\right),
$$

the geometric flux

$$
F_{a b}^{c}:=f_{a b}^{c}+\tilde{\partial}^{c} B_{a b}+\tilde{f}_{a}^{c m} B_{m b}+\tilde{f}_{b}^{c m} B_{a m}+\beta^{c m} H_{m a b}
$$

the non-geometric $Q$-flux

$$
\begin{aligned}
Q_{c}{ }^{a b}:= & \tilde{f}_{c}^{a b}+\partial_{c} \beta^{a b}+f_{c m}^{a} \beta^{m b}+f_{c m}^{b} \beta^{a m} \\
& +B_{c m} \tilde{\partial}^{m} \beta^{a b}+2 \beta^{m[\underline{a}} \tilde{\partial}^{\underline{b}]} B_{m c}+2 B_{c m} \tilde{f}_{n}^{m[\underline{a}} \beta^{\underline{b}] n}+2 \beta^{m[\underline{a}} \tilde{f}_{c}^{\underline{b}] n} B_{m n} \\
& +\beta^{a m} \beta^{b n} H_{m n c}
\end{aligned}
$$

and the non-geometric $R$-flux

$$
\begin{aligned}
R^{a b c}:= & 3\left(\tilde{\partial}^{[\underline{a}} \beta^{\underline{b c}]}+\tilde{f}_{m}^{[\underline{a b}} \beta^{\underline{c}] m}\right)+3\left(\beta^{[\underline{a} m} \partial_{m} \beta^{\underline{b c]}}+\beta^{[\underline{a} m} \beta^{\underline{\underline{b n}}} f^{\underline{c}]} m\right) \\
& +3\left(B_{m n} \beta^{[\underline{a} m} \tilde{\partial}^{n} \beta^{\underline{b c}]}+\beta^{[\underline{a} m} \beta^{\underline{\underline{b}} n} \tilde{\partial}^{\underline{c}]} B_{m n}+2 \beta^{[\underline{a} m} \beta^{\underline{\underline{b} n}} \tilde{f}_{\left[\underline{m}^{\underline{c}}\right.} B_{k \underline{n}]}\right) \\
& +\beta^{a m} \beta^{b n} \beta^{c p} H_{m n p} .
\end{aligned}
$$

Here we have used the strong constraint (2.22). We observe that, even in a holonomic frame, all four types of fluxes receive contributions. We have the $H$-flux

$$
H_{i j k}:=3 \partial_{[\underline{\underline{i}}} B_{\underline{j k}]}+3 B_{[\underline{i m}} \tilde{\partial}^{m} B_{\underline{j k}]},
$$

the geometric flux

$$
F_{i j}^{k}:=\tilde{\partial}^{k} B_{i j}+\beta^{k m} H_{m i j}
$$

the non-geometric $Q$-flux

$$
Q_{k}^{i j}:=\partial_{k} \beta^{i j}+B_{k m} \tilde{\partial}^{m} \beta^{i j}+2 \beta^{m[\underline{i} \tilde{\partial}} \underline{j}^{i} B_{m k}+\beta^{i m} \beta^{j n} H_{m n k}
$$

and the non-geometric $R$-flux

$$
\begin{aligned}
R^{i j k}:= & 3 \tilde{\partial}^{[\underline{i}} \beta^{\underline{j k}]}+3 \beta^{[\underline{i} m} \partial_{m} \beta \underline{j k]}+3 B_{m n} \beta^{[\underline{\underline{i} m}} \tilde{\partial}^{n} \beta^{\underline{j k}]}+3 \beta^{[\underline{\underline{i} m}} \beta^{\underline{j} n} \tilde{\partial}^{\underline{k}]} B_{m n} \\
& +\beta^{i m} \beta^{j n} \beta^{k p} H_{m n p} .
\end{aligned}
$$

The Jacobi-identities for the brackets (2.33) are trivial identities for the fluxes and can therefore be considered as their Bianchi identities. Again employing the 
strong constraint, one arrives at the five independent relations

$$
\begin{aligned}
& \mathcal{D}_{[\underline{a}} H_{\underline{b c d]}}-\frac{3}{2} H_{m[\underline{a b}} F^{m}{ }_{\underline{c d}]}=0 \\
& -\frac{1}{3} \tilde{\mathcal{D}}^{d} H_{a b c}+\mathcal{D}_{[\underline{a}} F_{\underline{b c}]}^{d}+F_{[\underline{b c}}^{m} F_{\underline{a}] m}^{d}+H_{m[\underline{a b}} Q_{\underline{c}]}{ }^{m d}=0
\end{aligned}
$$

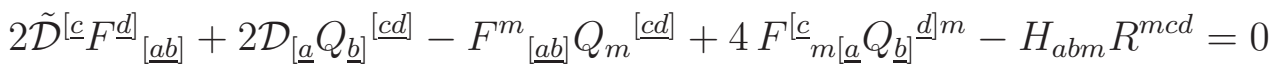

$$
\begin{aligned}
& -\frac{1}{3} \mathcal{D}_{d} R_{a b c}+\tilde{\mathcal{D}}^{[\underline{a}} Q_{d} \underline{\underline{b c}]}+Q_{m}{ }^{[\underline{b c}} Q_{d}{ }^{\underline{a}] m}+R^{m[\underline{a b}} F^{\underline{c}]}{ }_{m d}=0 \\
& \tilde{\mathcal{D}}^{[\underline{a}} R^{\underline{b c d}]}-\frac{3}{2} R^{m[\underline{a b}} Q_{m} \underline{\underline{c d}]}=0 .
\end{aligned}
$$

These relations constitute the generalization of the fluxes and their Bianchi identities to DFT.

\subsection{Coexistence of fluxes for orientifolds}

In the previous derivation we have solely employed the strong constraint but did not use any further constraint on the $B$-field and the $\beta$-field. Clearly, in a given patch string theory requires that only half of the degrees of freedom of these fields field can be independent. In addition, in the ten-dimensional theory one would require the strong constraint to be satisfied.

First we notice that, for tadpole cancellation, we need to perform an orientifold projection so that an interesting question to ask is which orientifold even components of the four types of fluxes can be turned on simultaneously. The details also depend on the concrete orientifold projection. Thus, let us first consider the behavior of the fields and fluxes under the world-sheet parity transformation $\Omega:(\sigma, \tau) \rightarrow(-\sigma, \tau)$. Here, the metric is invariant whereas the two-form $B$ and the bi-vector $\beta$ are anti-invariant. Moreover, since $\Omega$ maps the winding number to its inverse, this should also hold for the winding coordinate and the corresponding partial derivative. Thus, we have

$$
\Omega:\left\{\begin{array}{l}
\partial_{a} \rightarrow \partial_{a}, \quad \tilde{\partial}^{a} \rightarrow-\tilde{\partial}^{a} \\
B_{a b} \rightarrow-B_{a b}, \quad \beta^{a b} \rightarrow-\beta^{a b} \\
f_{b c}^{a} \rightarrow f_{b c}^{a}, \quad \tilde{f}_{a}^{b c} \rightarrow-\tilde{f}_{a}^{b c}
\end{array}\right.
$$

so that the fluxes transform as

$$
\Omega:\left\{\begin{array}{l}
H_{a b c} \rightarrow-H_{a b c} \\
F_{b c}^{a} \rightarrow F^{a} b c \\
Q_{a}^{b c} \rightarrow-Q_{a}^{b c} \\
R^{a b c} \rightarrow R^{a b c}
\end{array}\right.
$$

Thus, under $\Omega$ only the fluxes $F$ and $R$ are even. Dressing $\Omega$ by some $\mathbb{Z}_{2}$ spacetime symmetries introduces some extra minus signs along certain (reflected) legs, but is not expected to alter the general structure of the number of orientifold even fluxes to be turned on simultaneously. 
As it will not affect the computation performed in the next section, a complete analysis about which fluxes can be simultaneously turned on in a true string theory vacuum is beyond the scope of this paper. This question has been under debate recently, where for instance it has been pointed out that by a ScherkSchwarz reduction of DFT [18, 19, 20, 21] all fluxes can appear, but at the expense of weakening the strong constraint (2.22). Let us emphasize again that the analysis in the next section goes through without imposing any extra constraint on the fluxes beyond their Bianchi identities.

\section{Dimensional oxidation}

In this section we will start with the type IIA four-dimensional superpotential (2.10) and analyze its ten-dimensional origin. Concretely, as in [22] we will consider a toroidal orientifold for which we turn on all orientifold even geometric and non-geometric NS-NS fluxes, as well as all R-R fluxes. Computing the resulting four-dimensional scalar potential via Mathematica, in a step by step procedure we will inspect the underlying ten-dimensional terms, whose dimensional reduction leads to precisely the terms present in that scalar potential. This tedious analysis gets complicated by the presence of the Bianchi identities and tadpole conditions, which have to be invoked heavily during the course of the computation.

Let us emphasize that in this approach one has to clearly distinguish objects with background values (like vacuum expectation values for the fluxes) from objects that are moduli of the unfluxed Calabi-Yau compactification (like complex structure and Kähler moduli). We will treat the former as generic constant parameters that are only constrained by the Bianchi identities. Thus, we are not imposing any of the possible DFT constraints discussed in the previous section. We allow all of them to be present at the same time. Contrarily, for the initial compactification on the torus, we are working in the $\tilde{\partial}_{i}=0$ DFT frame with the generalized metric parametrized by $(G, B)$. As we will see, this mixed approach allows us to detect non-trivial terms in the ten-dimensional action that go beyond the usual supergravity action (2.2).

\subsection{A type IIA orientifold}

As our example we choose the familiar type IIA $\Omega I_{3}(-1)^{F_{L}}$ orientifold on the orbifold $T^{6} / \mathbb{Z}_{2} \times \mathbb{Z}_{2}$. Introducing on $T^{6}$ the coordinates

$$
z^{1}=R^{1} x^{1}+i R^{2} x^{2}, \quad z^{2}=R^{3} x^{3}+i R^{4} x^{4}, \quad z^{3}=R^{5} x^{5}+i R^{6} x^{6},
$$

where $0 \leq x^{i} \leq 1$ and $R^{i}$ denote the circumference of the $i$-th circle. The two $\mathbb{Z}_{2}$ actions are

$$
\begin{gathered}
\theta:\left(z^{1}, z^{2}, z^{3}\right) \rightarrow\left(-z^{1},-z^{2}, z^{3}\right) \\
\bar{\theta}:\left(z^{1}, z^{2}, z^{3}\right) \rightarrow\left(z^{1},-z^{2},-z^{3}\right),
\end{gathered}
$$


while $I_{3}$ acts as

$$
I_{3}:\left(z^{1}, z^{2}, z^{3}\right) \rightarrow\left(-\bar{z}^{1},-\bar{z}^{2},-\bar{z}^{3}\right) .
$$

The Hodge numbers of the $T^{6} / \mathbb{Z}_{2} \times \mathbb{Z}_{2}$ orbifold are $\left(h^{21}, h^{11}\right)=(3,51)$, but here we are only considering the untwisted sector with $\left(h_{\mathrm{ut}}^{21}, h_{\mathrm{ut}}^{11}\right)=(3,3)$. Due to the two $\mathbb{Z}_{2}$ symmetries, the $T^{6}$ splits into a product of three $T^{2}$ tori, i.e. in the untwisted sector of the orbifold we get three complex structure and three Kähler moduli, i.e. one pair for each $T^{2}$ factor

$$
\begin{aligned}
& \hat{u}_{1}=R^{1} / R^{2}, \quad \hat{u}_{2}=R^{3} / R^{4}, \quad \hat{u}_{3}=R^{5} / R^{6} \\
& t_{1}=R^{1} R^{2}, \quad t_{2}=R^{3} R^{4}, \quad t_{3}=R^{5} R^{6} \text {. }
\end{aligned}
$$

Let us choose the following basis of closed three-forms

$$
\begin{array}{ll}
\alpha_{0}=d x^{1} \wedge d x^{3} \wedge d x^{5}, & \beta^{0}=d x^{2} \wedge d x^{4} \wedge d x^{6}, \\
\alpha_{1}=d x^{1} \wedge d x^{4} \wedge d x^{6}, & \beta^{1}=d x^{2} \wedge d x^{3} \wedge d x^{5}, \\
\alpha_{2}=d x^{2} \wedge d x^{3} \wedge d x^{6}, & \beta^{2}=d x^{1} \wedge d x^{4} \wedge d x^{5}, \\
\alpha_{3}=d x^{2} \wedge d x^{4} \wedge d x^{5}, & \beta^{3}=d x^{1} \wedge d x^{3} \wedge d x^{6}
\end{array}
$$

satisfying $\int \alpha_{I} \wedge \beta^{J}=-\delta_{I}^{J}$. The holomorphic three-form is $\Omega_{3}=d z^{1} \wedge d z^{2} \wedge d z^{3}$ so that we can expand

$$
\begin{aligned}
\Omega^{c} & =\operatorname{Re}\left(i e^{-\phi} \Omega_{3}\right)-i C^{(3)} \\
& =S \beta^{0}-U_{1} \beta^{1}-U_{2} \beta^{2}-U_{3} \beta^{3},
\end{aligned}
$$

where

$$
\begin{aligned}
S & =e^{-\phi} R^{2} R^{4} R^{6}-i C_{246}^{(3)}, & U_{1} & =e^{-\phi} R^{2} R^{3} R^{5}+i C_{235}^{(3)}, \\
U_{2} & =e^{-\phi} R^{1} R^{4} R^{5}+i C_{145}^{(3)}, & U_{3} & =e^{-\phi} R^{1} R^{3} R^{6}+i C_{136}^{(3)}
\end{aligned}
$$

are the bosonic components of the chiral superfields for the orientifold even complex structure moduli. The chiral superfields for the complexified Kähler moduli are

$$
T_{1}=t_{1}+i B_{12}, \quad T_{2}=t_{2}+i B_{34}, \quad T_{3}=t_{3}+i B_{56}
$$

Then, the non-vanishing components of the internal ten-dimensional metric in string frame are

$$
g_{M N}=\operatorname{blockdiag}\left(\frac{e^{2 \phi}}{t_{1} t_{2} t_{3}} \tilde{g}_{\mu \nu},\left(R^{1}\right)^{2},\left(R^{2}\right)^{2},\left(R^{3}\right)^{2},\left(R^{4}\right)^{2},\left(R^{5}\right)^{2},\left(R^{6}\right)^{2}\right)
$$


where here $\tilde{g}_{\mu \nu}$ denotes the four-dimensional metric in Einstein-frame. The internal components can be expressed as

$$
\begin{array}{lll}
g_{11}=t_{1} \sqrt{\frac{u_{2} u_{3}}{s u_{1}}}, & g_{22}=t_{1} \sqrt{\frac{s u_{1}}{u_{2} u_{3}}}, & g_{33}=t_{2} \sqrt{\frac{u_{1} u_{3}}{s u_{2}}} \\
g_{44}=t_{2} \sqrt{\frac{s u_{2}}{u_{1} u_{3}}}, & g_{55}=t_{3} \sqrt{\frac{u_{2} u_{1}}{s u_{3}}}, & g_{66}=t_{3} \sqrt{\frac{s u_{3}}{u_{2} u_{1}}}
\end{array}
$$

where $s$ and the $u_{i}$ are the real components of the complex fields in (3.7). The tree-level Kähler potential for the seven moduli fields is given by

$$
K=-\ln \left(\frac{S+\bar{S}}{2}\right)-\sum_{i=1,2,3} \ln \left(\frac{U_{i}+\overline{U_{i}}}{2}\right)-\sum_{i=1,2,3} \ln \left(\frac{T_{i}+\overline{T_{i}}}{2}\right) .
$$

The independent, orientifold even components of the NS-NS background fluxes are

$$
\begin{array}{ll}
\bar{H}_{i j k}: & \bar{H}_{135}, \bar{H}_{146}, \bar{H}_{236}, \bar{H}_{245} \\
\bar{F}_{i j}^{k}: & \bar{F}_{13}^{6}, \bar{F}_{23}^{5}, \bar{F}_{24}^{6}, \bar{F}_{14}^{5} \\
& \bar{F}_{35}^{2}, \bar{F}_{45}^{1}, \bar{F}_{46}^{2}, \bar{F}_{36}^{1} \\
& \bar{F}_{51}^{4}, \bar{F}_{61}^{3}, \bar{F}_{62}^{4}, \bar{F}_{52}^{3} \\
\bar{Q}_{k}{ }^{i j}: & \bar{Q}_{1}^{35}, \bar{Q}_{2}^{45}, \bar{Q}_{1}^{46}, \bar{Q}_{2}^{36} \\
& \bar{Q}_{5}^{13}, \bar{Q}_{6}^{23}, \bar{Q}_{5}^{24}, \bar{Q}_{6}^{14} \\
& \bar{Q}_{3}{ }^{51}, \bar{Q}_{4}{ }^{61}, \bar{Q}_{3}^{62}, \bar{Q}_{4}{ }^{52} \\
\bar{R}^{i j k}: & \bar{R}^{246}, \bar{R}^{235}, \bar{R}^{145}, \bar{R}^{136} .
\end{array}
$$

In the R-R sector the orientifold even fluxes are

$$
\bar{G}^{(0)}, \bar{G}_{12}^{(2)}, \bar{G}_{34}^{(2)}, \bar{G}_{56}^{(2)}, \bar{G}_{1234}^{(4)}, \bar{G}_{1256}^{(4)}, \bar{G}_{3456}^{(4)}, \bar{G}_{123456}^{(6)} .
$$

The detailed form of the superpotential

$$
W_{\mathrm{NS}}=-\frac{i}{4} \int_{X} \overline{\mathfrak{H}}^{C} \wedge \Omega^{c}+\frac{1}{4} \int_{X} e^{i J_{c}} \wedge \bar{G}
$$

in terms of the fluxes and moduli can be found in appendix $\mathrm{A}$

\subsection{Oxidation to $10 \mathrm{D}$ type IIA action}

Taking now the superpotential (A.2) and computing the scalar F-term potential, the procedure described in the beginning of section 3 reveals that the result can be obtained from a couple of generalized kinetic terms in a ten-dimensional action

$$
S=\frac{1}{2} \int d^{10} x \sqrt{-g}\left(\mathcal{L}_{1}^{\mathrm{NS}}+\mathcal{L}_{2}^{\mathrm{NS}}+\mathcal{L}^{\mathrm{R}}\right)
$$


In order to express the result it turns out to be convenient to introduce the following combinations or orbits of fluxes

$$
\begin{aligned}
\mathfrak{H}_{i j k} & =\bar{H}_{i j k}+3 \bar{F}^{m}{ }_{[i \underline{j}} B_{m \underline{k}]}+3 \bar{Q}_{[\underline{i}}^{m n} B_{m \underline{j}} B_{n \underline{k}]}+\bar{R}^{m n p} B_{m[\underline{i}} B_{n \underline{j}} B_{p \underline{k}]} \\
\mathfrak{F}_{j k}^{i} & =\bar{F}^{i}{ }_{j k}+2 \bar{Q}_{[\underline{j}}^{m i} B_{m \underline{k}]}+\bar{R}^{m n i} B_{m[\underline{j}} B_{n \underline{k}]} \\
\mathfrak{Q}_{k}{ }^{i j} & =\bar{Q}_{k}{ }^{i j}+\bar{R}^{m i j} B_{m k} \\
\mathfrak{R}^{i j k} & =\bar{R}^{i j k} .
\end{aligned}
$$

Here by the overlining we indicated which fields in the dimensional reduction are treated as backgrounds and which as moduli. Then, we oxidize a term containing three metric factors

$$
\begin{aligned}
\mathcal{L}_{1}^{\mathrm{NS}}=-\frac{e^{-2 \phi}}{12}( & \mathfrak{H}_{i j k} \mathfrak{H}_{i^{\prime} j^{\prime} k^{\prime}} g^{i i^{\prime}} g^{j j^{\prime}} g^{k k^{\prime}}+3 \mathfrak{F}^{i}{ }_{j k} \mathfrak{F}^{i^{\prime}}{ }_{j^{\prime} k^{\prime}} g_{i i^{\prime}} g^{j j^{\prime}} g^{k k^{\prime}} \\
& \left.+3 \mathfrak{Q}_{k}{ }^{i j} \mathfrak{Q}_{k^{\prime}}{ }^{i^{\prime} j^{\prime}} g_{i i^{\prime}} g_{j j^{\prime}} g^{k k^{\prime}}+\mathfrak{R}^{i j k} \mathfrak{R}^{i^{\prime} j^{\prime} k^{\prime}} g_{i i^{\prime}} g_{j j^{\prime}} g_{k k^{\prime}}\right)
\end{aligned}
$$

and a term containing a single metric factor

$$
\begin{aligned}
\mathcal{L}_{2}^{\mathrm{NS}}=-\frac{e^{-2 \phi}}{2}\left(\mathfrak{F}_{n i}^{m} \mathfrak{F}^{n}{ }_{m i^{\prime}} g^{i i^{\prime}}+\mathfrak{Q}_{m}{ }^{n i} \mathfrak{Q}_{n}{ }^{m i^{\prime}} g_{i i^{\prime}}\right. \\
\\
\left.\quad-\mathfrak{H}_{m n i} \mathfrak{Q}_{i^{\prime}}{ }^{m n} g^{i i^{\prime}}-\mathfrak{F}_{m n}^{i} \mathfrak{R}^{m n i^{\prime}} g_{i i^{\prime}}\right) .
\end{aligned}
$$

The contribution from the Ramond-Ramond-sector is

$$
\mathcal{L}^{\mathrm{R}}=-\frac{1}{2} \sum_{p=0,2,4,6}\left|G^{(p)}\right|^{2},
$$

where the $p$-form field strengths are defined as

$$
G^{(p)}=\frac{1}{p !} G_{i_{1} \ldots i_{p}}^{(p)} d x^{i_{1}} \wedge \ldots \wedge d x^{i_{p}}
$$

with the components

$$
\begin{aligned}
& G^{(0)}=\bar{G}^{(0)}+\frac{1}{6} \mathfrak{R}^{m n p} C_{m n p}^{(3)} \\
& G_{i j}^{(2)}=\bar{G}_{i j}^{(2)}-B_{i j} \bar{G}^{(0)}+\mathfrak{Q}_{[\underline{i}}^{m n} C_{m n \underline{j}]}^{(3)} \\
& G_{i j k l}^{(4)}=\bar{G}_{i j k l}^{(4)}-6 B_{[\underline{i j}} \bar{G}_{\underline{k l}]}^{(2)}+3 B_{[\underline{i j}} B_{\underline{k l}]} \bar{G}^{(0)}-6 \mathfrak{F}^{m}{ }_{[\underline{i j}} C_{m \underline{k l}]}^{(3)} \\
& G_{i j k l m n}^{(6)}=\bar{G}_{i j k l m n}^{(6)}-15 B_{[\underline{i j}} \bar{G}_{\underline{k l m n}]}^{(4)}+45 B_{[\underline{i j}} B_{\underline{k l}} \bar{G}_{\underline{m n}]}^{(2)} \\
& -15 B_{[\underline{i j}} B_{\underline{k l}} B_{\underline{m n}]} \bar{G}^{(0)}-20 \mathfrak{H}_{[\underline{i j k}} C_{\underline{l m n}]}^{(3)} .
\end{aligned}
$$


Here we included the background flux $G=e^{-B} \bar{G}$. Taking now the action (3.15) and dimensionally reducing it to four-dimensions gives a scalar potential which is a sum of the desired F-term and a D-term. Note that the F-term scalar potential has several additional terms, which upon invoking the Bianchi identities for constant fluxes

$$
\begin{aligned}
& \bar{H}_{m[\underline{a b}} \bar{F}_{\underline{c d}]}^{m}=0 \\
& \bar{F}_{[\underline{b c}}^{m} \bar{F}_{\underline{a}] m}^{d}+\bar{H}_{m[\underline{a b}} \bar{Q}_{\underline{c}]}^{m d}=0 \\
& \bar{F}_{[\underline{[a b}]}^{m} \bar{Q}_{m}{ }^{[\underline{c d}]}-4 \bar{F}_{m[\underline{a}}^{\left[\underline{c}_{\underline{b}}{ }^{\underline{d}] m}\right.} \overline{\bar{H}}_{m a b} \bar{R}^{m c d}=0 \\
& \bar{Q}_{m}{ }^{[b c} \bar{Q}_{d}{ }^{\underline{a}] m}+\bar{R}^{m[\underline{a b}} \bar{F}_{m d}^{\underline{c}]}=0 \\
& \bar{R}^{m[\underline{a b}} \bar{Q}_{m} \underline{c d]}=0 .
\end{aligned}
$$

are nullified. More details on this computation are presented in appendix B.

In order to describe the D-term let us define a three-form

$$
\tau=\frac{1}{6} \tau_{i j k} d x^{i} \wedge d x^{j} \wedge d x^{k}
$$

with

$$
\tau_{i j k}=\bar{H}_{i j k} \bar{G}^{(0)}+3 \bar{F}_{[\underline{i j}}^{m} \bar{G}_{m \underline{k}]}^{(2)}-\frac{3}{2} \bar{Q}_{[\underline{i}}^{m n} \bar{G}_{m n \underline{j k}]}^{(4)}-\frac{1}{6} \bar{R}^{m n p} \bar{G}_{m n p i j k}^{(6)} .
$$

Then the D-term is

$$
V_{D}=-\frac{1}{2} e^{K} t_{1} t_{2} t_{3}\left[s \tau_{135}-u_{1} \tau_{146}-u_{2} \tau_{236}-u_{3} \tau_{245}\right]
$$

which is a contribution to the NS-NS tadpole. As in [22], due to R-R tadpole cancellation we expect this term to be canceled against the tensions of the D6branes and O6-planes. From this one can deduce the existence of a Chern-Simons term

$$
S_{\mathrm{CS}} \sim \int C^{(7)} \wedge \tau
$$

in the ten-dimensional action, which was also suggested in [8].

\subsection{A type IIB orientifold}

As a second explicit example, we now perform the analogous computation for a $\mathrm{T}$ dual type IIB orientifold. Applying a T-duality in the three-directions $x_{2}, x_{4}, x_{6}$, leads to the type IIB orientifold with action $\Omega I_{6}(-1)^{F_{L}}$ on the orbifold $T^{6} / \mathbb{Z}_{2} \times$ $\mathbb{Z}_{2}$. The fixed point set of this orientifold gives $03 / O 7$-planes. The complex coordinates $z_{i}$ 's on $T^{6}=T^{2} \times T^{2} \times T^{2}$ are defined as

$$
z^{1}=x^{1}+i U_{1} x^{2}, \quad z^{2}=x^{3}+i U_{2} x^{4}, \quad z^{3}=x^{5}+i U_{3} x^{6},
$$


where the three complex structure moduli $U_{i}$ 's can be written as $U_{i}=u_{i}+i v_{i}, i=$ $1,2,3$. The orbifold symmetry of the two $\mathbb{Z}_{2}$ acts as in (3.2) while the involution $I_{6}$ is

$$
I_{6}:\left(z^{1}, z^{2}, z^{3}\right) \rightarrow\left(-z^{1},-z^{2},-z^{3}\right) .
$$

Choosing the basis of closed three-forms (3.5), the holomorphic 3-form $\Omega_{3}$ can be expanded as

$$
\begin{aligned}
\Omega_{3}=\alpha_{0}+i\left(U_{1} \beta^{1}+\right. & \left.U_{2} \beta^{2}+U_{3} \beta^{3}\right)-i U_{1} U_{2} U_{3} \beta^{0} \\
& -U_{2} U_{3} \alpha_{1}-U_{1} U_{3} \alpha_{2}-U_{1} U_{2} \alpha_{3} .
\end{aligned}
$$

In addition one has the axio-dilaton chiral superfield, whose bosonic component is

$$
S=e^{-\phi}-i C^{(0)} .
$$

The chiral superfields related to the Kähler moduli are generically encoded in the complexified four-cycle volumes

$$
J^{c}=\frac{1}{2} e^{-\phi} J \wedge J+i C^{(4)} .
$$

In our case, these moduli are

$$
T_{1}=\tau_{1}+i C_{3456}^{(4)}, \quad T_{2}=\tau_{2}+i C_{1256}^{(4)}, \quad T_{3}=\tau_{3}+i C_{1234}^{(4)},
$$

where the real parts can be expressed in terms of the two-cycle volumes $t_{i}$ as $\tau_{1}=e^{-\phi} t_{2} t_{3}, \tau_{2}=e^{-\phi} t_{3} t_{1}$ and $\tau_{3}=e^{-\phi} t_{1} t_{2}$. We also need to express the two-cycle volumes $t_{i}$ in terms of the four-cycles volumes $\tau_{i}$

$$
t_{1}=\sqrt{\frac{\tau_{2} \tau_{3}}{\tau_{1} s}}, \quad t_{2}=\sqrt{\frac{\tau_{1} \tau_{3}}{\tau_{2} s}}, \quad t_{3}=\sqrt{\frac{\tau_{1} \tau_{2}}{\tau_{3} s}}
$$

with $s=\operatorname{Re}(S)$. Now, the non-vanishing components of the metric in string frame are

$$
g_{M N}=\operatorname{blockdiag}\left(\frac{e^{\frac{\phi}{2}}}{\sqrt{\tau_{1} \tau_{2} \tau_{3}}} \tilde{g}_{\mu \nu}, g_{i j}\right) .
$$

Further, the string frame internal metric $g_{i j}$ is also block-diagonal and has the following non-vanishing components,

$$
\begin{aligned}
& g_{11}=\frac{t_{1}}{u_{1}}, \quad g_{12}=-\frac{t_{1} v_{1}}{u_{1}}=g_{21}, \quad g_{22}=\frac{t_{1}\left(u_{1}^{2}+v_{1}^{2}\right)}{u_{1}}, \\
& g_{33}=\frac{t_{2}}{u_{2}}, \quad g_{34}=-\frac{t_{2} v_{2}}{u_{2}}=g_{43}, \quad g_{44}=\frac{t_{2}\left(u_{2}^{2}+v_{2}^{2}\right)}{u_{2}} \\
& g_{55}=\frac{t_{3}}{u_{3}},
\end{aligned}
$$


Using (3.33) all components can also be expressed in term of the non-axionic components of the seven chiral superfields $S, T_{1,2,3}, U_{1,2,3}$.

Since the background fluxes $F^{i}{ }_{j k}$ and $R^{i j k}$ are odd under the orientifold projection, the only invariant fluxes are the following components of the three-forms $H$ and $G^{(3)}$

$$
\begin{aligned}
\bar{H}: & \bar{H}_{135}, \bar{H}_{146}, \bar{H}_{236}, \bar{H}_{245} \\
& \bar{H}_{246}, \bar{H}_{235}, \bar{H}_{145}, \bar{H}_{136}, \\
\bar{G}^{(3)}: & \bar{G}_{135}, \bar{G}_{146}, \bar{G}_{236}, \bar{G}_{245} \\
& \bar{G}_{246}, \bar{G}_{235}, \bar{G}_{145}, \bar{G}_{136}
\end{aligned}
$$

and the components of non-geometric $Q$-flux 5

$$
\begin{aligned}
\bar{Q}: & \bar{Q}_{1}{ }^{35}, \bar{Q}_{2}{ }^{45}, \bar{Q}_{1}{ }^{46}, \bar{Q}_{2}{ }^{36} \\
& \bar{Q}_{5}{ }^{13}, \bar{Q}_{6}{ }^{23}, \bar{Q}_{5}{ }^{24}, \bar{Q}_{6}{ }^{14} \\
& \bar{Q}_{3}{ }^{51}, \bar{Q}_{4}{ }^{61}, \bar{Q}_{3}{ }^{62}, \bar{Q}_{4}{ }^{52} \\
& \bar{Q}_{2}{ }^{35}, \bar{Q}_{5}{ }^{23}, \bar{Q}_{3}{ }^{52}, \bar{Q}_{2}{ }^{46} \\
& \bar{Q}_{4}{ }^{51}, \bar{Q}_{1}{ }^{45}, \bar{Q}_{5}{ }^{14}, \bar{Q}_{4}{ }^{62} \\
& \bar{Q}_{6}{ }^{13}, \bar{Q}_{3}{ }^{61}, \bar{Q}_{1}{ }^{36}, \bar{Q}_{6}{ }^{24}
\end{aligned}
$$

The full superpotential is given by

$$
W=-\frac{i}{4} \int_{X}\left(S \bar{H}+\bar{Q} \cdot J^{c}\right) \wedge \Omega_{3}+\frac{1}{4} \int_{X} \bar{G}^{(3)} \wedge \Omega_{3}=W_{N S}+W_{R}
$$

where the three-form $\bar{Q} \cdot J^{c}=\frac{1}{6}\left(\bar{Q} \cdot J^{c}\right)_{i j k} d x^{i} \wedge d x^{j} \wedge d x^{k}$ is defined as

$$
\left(\bar{Q} \cdot J^{c}\right)_{i j k}=\frac{3}{2} \bar{Q}_{[\underline{i}}^{m n} J_{m n \underline{j k]}}^{c} .
$$

The explicit form of this superpotential is presented in appendix A. Together with the Kähler potential

$$
K=-\ln \left(\frac{S+\bar{S}}{2}\right)-\sum_{i=1,2,3} \ln \left(\frac{U_{i}+\overline{U_{i}}}{2}\right)-\sum_{i=1,2,3} \ln \left(\frac{T_{i}+\overline{T_{i}}}{2}\right)
$$

it allows now to compute the effective four-dimensional scalar potential.

\footnotetext{
${ }^{5}$ For S-dual completion of type IIB orientifolds, one needs to introduce additional nongeometric P-fluxes which are R-R analogue of non-geometric Q-fluxes [23].
} 


\subsection{Oxidation to 10D type IIB action}

Similar to the previous type IIA case, a close inspection of the resulting scalar potential reveals that it can be obtained via dimensional reduction from a couple of generalized kinetic terms in a ten-dimensional action

$$
S=\frac{1}{2} \int d^{10} x \sqrt{-g}\left(\mathcal{L}_{1}^{\mathrm{NS}}+\mathcal{L}_{2}^{\mathrm{NS}}+\mathcal{L}^{\mathrm{R}}\right)
$$

with the oxidized terms given as

$$
\begin{aligned}
\mathcal{L}_{1}^{\mathrm{NS}} & =-\frac{e^{-2 \phi}}{12}\left(\bar{H}_{i j k} \bar{H}_{i^{\prime} j^{\prime} k^{\prime}} g^{i i^{\prime}} g^{j j^{\prime}} g^{k k^{\prime}}+3 \bar{Q}_{k}^{i j} \bar{Q}_{k^{\prime}}^{i^{\prime} j^{\prime}} g_{i i^{\prime}} g_{j j^{\prime}} g^{k k^{\prime}}\right) \\
\mathcal{L}_{2}^{\mathrm{NS}} & =-\frac{e^{-2 \phi}}{2}\left(\bar{Q}_{m}{ }^{n i} \bar{Q}_{n}{ }^{m i^{\prime}} g_{i i^{\prime}}-\bar{H}_{m n i} \bar{Q}_{i^{\prime}}{ }^{m n} g^{i i^{\prime}}\right) \\
\mathcal{L}^{\mathrm{R}} & =-\frac{1}{12} G_{i j k}^{(3)} G_{i^{\prime} j^{\prime} k^{\prime}}^{(3)} g^{i i^{\prime}} g^{j j^{\prime}} g^{k k^{\prime}}
\end{aligned}
$$

with

$$
G_{i j k}^{(3)}=\bar{G}_{i j k}-C^{(0)} \bar{H}_{i j k}+\frac{3}{2} \bar{Q}_{[\underline{i}}^{l m} C_{l m \underline{j k]}}^{(4)} .
$$

Let us mention that for our example there are no two-forms anti-invariant under the orientifold projection so that no $B_{2}$ and $C_{2}$ moduli are present. As a consequence, the type IIA flux orbits like $\mathfrak{H}_{i j k}$ and $\mathfrak{Q}_{k}{ }^{i j}$ simplify to $H_{i j k}$ and $Q_{k}{ }^{i j}$. The redefined three-form (3.43) also appeared in [58].

As for the type IIA case, invoking the Bianchi identities for constant fluxes (3.22), the dimensional reduction of the above kinetic terms leads to the F-term scalar potential induced by the superpotential and some additional D-terms. More details on this lengthy computation can be found in appendix B. There arises a D-term

$$
V_{D 3}=\frac{1}{2} e^{K} u_{1} u_{2} u_{3}\left[20 \bar{G}_{[\underline{123}} \bar{H}_{\underline{456}]} s\right]
$$

corresponding to the D3-brane tadpole and a D-term

$$
V_{D 7}=-\frac{1}{2} e^{K} u_{1} u_{2} u_{3}\left[\bar{Q}_{[\underline{1}}^{j k} \bar{G}_{j k \underline{2}]} \tau_{1}+\bar{Q}_{[\underline{3}}^{j k} \bar{G}_{j k \underline{4}]} \tau_{2}+\bar{Q}_{[\underline{5}}^{j k} \bar{G}_{j k \underline{6}]} \tau_{3}\right]
$$

corresponding to the three D7-brane tadpoles. Since these are related to the R-R tadpoles, there should also exist CS-terms

$$
S_{C S} \sim-\int C^{(4)} \wedge G \wedge H+\int C^{(8)} \wedge Q \cdot G,
$$

where the first one is familiar from the standard type IIB supergravity action. 


\section{Relation to DFT}

Let us recall that for both the type IIA and the type IIB orientifold we have succeeded to extract ten-dimensional actions (3.15) and (3.41), whose dimensional reduction induces precisely the F-term scalar potential implied by the flux induced superpotential and additional D-term potentials, which are related to the tadpole cancellation conditions. Thus, the program of the derivation of the Gukov-VafaWitten superpotential first performed in [50] could be realized also for more generic fluxes. The question now is whether the ten-dimensional actions are really related to the main candidate for an action including both geometric and non-geometric fluxes, i.e. the DFT action (2.19). In this section, let us discuss this in more detail, where we consider the NS-NS and the R-R part separately.

\subsection{Relating the oxidized NS-NS action to DFT}

As in the discussions of Scherk-Schwarz reductions of DFT in [18, 19, 20, 46, 48, 49], it is convenient to introduce the DFT fluxes $\mathcal{F}_{I J K}$ with

$$
\mathcal{F}_{i j k}=H_{i j k}, \quad \mathcal{F}^{i}{ }_{j k}=F^{i}{ }_{j k}, \quad \mathcal{F}_{k}^{i j}=Q_{k}^{i j}, \quad \mathcal{F}^{i j k}=R^{i j k} .
$$

Then, we observe that the NS-NS sector action can be compactly written as

$$
\mathcal{L}_{1}^{\mathrm{NS}}+\mathcal{L}_{2}^{\mathrm{NS}}=e^{-2 \phi} \mathcal{F}_{I J K} \mathcal{F}_{I^{\prime} J^{\prime} K^{\prime}}\left(\frac{1}{4} \mathcal{H}^{I I^{\prime}} \eta^{J J^{\prime}} \eta^{K K^{\prime}}-\frac{1}{12} \mathcal{H}^{I I^{\prime}} \mathcal{H}^{J J^{\prime}} \mathcal{H}^{K K^{\prime}}\right)
$$

Note that the off-diagonal components of the generalized metric $\mathcal{H}$ automatically

generate those flux orbits (3.16) $\mathfrak{H}_{i j k}, \mathfrak{F}^{i}{ }_{j k}, \mathfrak{Q}_{k}{ }^{i j}$ and $\mathfrak{R}^{i j k}$. This form of the action is very reminiscent of the flux formulation of the DFT action, which in a flat frame can be expressed as follows

$$
\begin{aligned}
& S_{\mathrm{DFT}}=\frac{1}{2} \int d^{20} X e^{-2 d}\left[\mathcal { F } _ { A B C } \mathcal { F } _ { A ^ { \prime } B ^ { \prime } C ^ { \prime } } \left(\frac{1}{4} S^{A A^{\prime}} \eta^{B B^{\prime}} \eta^{C C^{\prime}}\right.\right. \\
&\left.-\frac{1}{12} S^{A A^{\prime}} S^{B B^{\prime}} S^{C C^{\prime}}-\frac{1}{6} \eta^{A A^{\prime}} \eta^{B B^{\prime}} \eta^{C C^{\prime}}\right) \\
&\left.+\mathcal{F}_{A} \mathcal{F}_{A^{\prime}}\left(\eta^{A A^{\prime}}-S^{A A^{\prime}}\right)\right] .
\end{aligned}
$$

Here $\mathcal{F}_{A}$ is defined as

$$
\mathcal{F}_{A}=\Omega^{B}{ }_{B A}+2 E_{A}^{I} \partial_{I} d
$$

with the generalized Weitzenböck connection

$$
\Omega_{A B C}=E_{A}^{I} \partial_{I} E_{B}^{J} E_{C J} .
$$


In this form the action is motivated by the scalar potential appearing in gauged supergravity. In performing the dimensional reduction of (4.3), we allow generic constant background fluxes $\overline{\mathcal{F}}_{A B C}$. Moreover, for the dynamical fields we solve the strong constraint via $\tilde{\partial}^{i}=0$ and use the generalized non-holonomic frame (2.18). Then, one can compute for the components of $\mathcal{F}_{A B C}$

$$
\begin{aligned}
\mathcal{F}_{a b c} & =e_{a}{ }^{i} e_{b}{ }^{j} e_{c}{ }^{k} \mathfrak{H}_{i j k}, & \mathcal{F}^{a}{ }_{b c} & =e^{a}{ }_{i} e_{b}{ }^{j} e_{c}{ }^{k} \mathfrak{F}^{i}{ }_{j k}, \\
\mathcal{F}_{c}{ }^{a b} & =e^{a}{ }_{i} e^{b}{ }_{j} e_{c}{ }^{k} \mathfrak{Q}_{k}{ }^{i j}, & \mathcal{F}^{a b c} & =e^{a}{ }_{i} e^{b}{ }_{j} e^{c}{ }_{k} \mathfrak{R}^{i j k}
\end{aligned}
$$

showing that the flux orbits (3.16) arise automatically from the off-diagonal component of the generalized non-holonomic frame (2.18).

Thus, for type IIA the first two terms in (4.3) precisely match our oxidized NS-NS action (4.2). The additional three terms in (4.3) cannot be detected by our computation. On the one hand, the term $\mathcal{F}_{A B C} \mathcal{F}^{A B C}$ vanishes for orientifold even fluxes and, on the other hand, $\mathcal{F}_{A}$ vanishes as we only have fluxes with precisely one leg on each $T^{2}$ as well as constant metric and dilaton. We conclude that the oxidized type IIA NS-NS sector action is indeed compatible with the ten-dimensional DFT action in the NS-NS sector. The same holds for the type IIB orientifold, where the result is even simpler as all internal components of the B-field are modded out by the orientifold projection.

\subsection{Relating the oxidized R-R action to DFT}

Let us now investigate whether this continues to the R-R sector. In order to compactly write the oxidized action, as shown in [59, 60, 61, 62], it is convenient to put the R-R fields into the spinor representation of $O(10,10)$. One defines the generalized $\Gamma$-matrices as $\Gamma^{A}=\left(\Gamma_{a}, \Gamma^{a}\right)$ where we remind the reader that $a$ is a flat index. Then all $\Gamma^{A}$ can be chosen to be real and the Clifford algebra reads

$$
\left\{\Gamma^{A}, \Gamma^{B}\right\}=\eta^{A B}
$$

Therefore, the only non-vanishing anti-commutator is $\left\{\Gamma^{a}, \Gamma_{b}\right\}=\delta^{a}{ }_{b}$. Thus $\Gamma_{a}$ can be considered as a fermionic lowering operator and $\Gamma^{a}$ as the corresponding raising operator. Introducing a vacuum state $|0\rangle$ with $\Gamma_{a}|0\rangle=0$, we can put all $\mathrm{R}$ - $\mathrm{R}$ fields in the spinor representation as

$$
\mathcal{G}=\sum_{n} \frac{1}{n !} G_{i_{1} \ldots i_{n}}^{(n)} e_{a_{1}}{ }^{i_{1}} \ldots e_{a_{n}}{ }^{i_{n}} \Gamma^{a_{1} \ldots a_{n}}|0\rangle,
$$

where as usual $\Gamma^{a_{1} \ldots a_{n}}$ defines the totally anti-symmetrized product of $n \Gamma$ matrices. Similarly, we combine all the R-R gauge potentials $C^{(n)}$ into a spinor $\mathcal{C}$. Then, as shown in [19], one can compactly define the R-R field strengths as

$$
\mathcal{G}=\not \mathcal{C}
$$


with the generalized fluxed Dirac operator defined as

$$
\not=\Gamma^{A} D_{A}-\frac{1}{3} \Gamma^{A} \mathcal{F}_{A}-\frac{1}{6} \Gamma^{A B C} \mathcal{F}_{A B C}
$$

Evaluating this for the $G^{(3)}$-flux in the type IIB example, we find

$$
\begin{gathered}
\frac{1}{3 !} G_{i j k}^{(3)} e_{a}{ }^{i} e_{b}{ }^{j} e_{c}{ }^{k} \Gamma^{a b c}|0\rangle=\Gamma^{A} D_{A} \mathcal{C}-\frac{1}{6} \Gamma^{A B C} \mathcal{F}_{A B C} \mathcal{C} \\
=\frac{1}{2} \Gamma^{a} \partial_{a} C_{b c}^{(2)} \Gamma^{b c}|0\rangle-\frac{1}{6} H_{a b c} C^{(0)} \Gamma^{a b c}|0\rangle \\
\quad-\frac{1}{6} \frac{3}{4 !} \Gamma^{m} \Gamma_{n} \Gamma_{p} Q_{m}{ }^{n p} C_{i j k l}^{(4)} e_{a}^{i} e_{b}^{j} e_{c}{ }^{k} e_{d}^{l} \Gamma^{a b c d}|0\rangle \\
=\frac{1}{2} \partial_{i} C_{j k}^{(2)} e_{a}^{i} e_{b}^{j} e_{c}{ }^{k} \Gamma^{a b c}|0\rangle-\frac{1}{6} H_{i j k} C^{(0)} e_{a}{ }^{i} e_{b}^{j} e_{c}^{k} \Gamma^{a b c}|0\rangle \\
\quad+\frac{1}{6} \frac{3}{2} Q_{a}{ }^{m n} C_{i j k l}^{(4)} e_{b}^{i} e_{c}^{j} e_{m}{ }^{k} e_{n}{ }^{l} \Gamma^{a b c}|0\rangle,
\end{gathered}
$$

from which one can conclude6

$$
G_{i j k}^{(3)}=\bar{G}_{i j k}-C^{(0)} \bar{H}_{i j k}+\frac{3}{2} \bar{Q}_{[\underline{i}}^{l m} C_{l m \underline{j k]}}^{(4)} .
$$

This is precisely the oxidized relation (3.43). Using (4.6), the last term in (4.10) also reproduces precisely the type IIA NS-NS flux contributions in (3.21). Therefore, we conclude that the oxidized $\mathrm{R}$ - $\mathrm{R}$ action is compatible with the proposed DFT action, as well. We find it quite remarkable to realize how much information about the underlying ten-dimensional action is already contained in this innocently looking superpotential.

\section{Conclusions}

In this paper we have analyzed a couple of aspects of non-geometric flux compactifications of the type II string theories to four-dimensions in the light of recent progress in DFT. First, we have rederived the form of the four type of fluxes $H, F, Q$ and $R$ in terms of the fundamental fields appearing in the generalized metric of DFT. Since the latter are subject to a number of constraints, it appears to be a non-trivial question, which fluxes one can truly turn on in a string theory vacuum. This complication arises, as DFT in ten-dimensions contains too many degrees of freedom so that it has to be equipped with some version of a strong constraint.

In the main part of the paper, we have explicitly verified that the type IIA and type IIB superpotentials for constant geometric and non-geometric fluxes are compatible with the recently proposed form of the tree-level DFT action

\footnotetext{
${ }^{6}$ In (4.11) we were using that $f^{a}{ }_{b c}=0$, as it is odd under orientifold projection.
} 
in both the NS-NS and the R-R sector. We would like to emphasize that this result holds independent of the actual realization of the non-vanishing fluxes, i.e. prior to invoking any additional constraints beyond the strong constraint, which was employed for deriving the Bianchi identities. We were investigating two concrete toroidal orientifolds, for which we turned on constant orientifold even fluxes and then oxidized the resulting F-term scalar potential to ten-dimensions. This led to a number of kinetic terms for the fluxes, which showed precisely the intricate structure present in the flux DFT action, which was motivated by gauged supergravity. In this computation, the fluctuations of the massless fields $G$ and $B$ were still treated in the usual geometric frame, whereas for the backgrounds generic, i.e. also non-geometric, fluxes were allowed. This found precise match provides further compelling evidence for both the correctness of the superpotential and the DFT action.

Due to our still restricted ansatz, it was not possible to see all DFT terms. To also make them apparent, a generalization of our computation is necessary. Moreover, it would be interesting to generalize our explicit computation to general Calabi-Yau threefolds with some background fluxes turned on. It would also be interesting, for M-theory compactifications on $G_{2}$ manifolds, to generalize the computation of [47] to include also non-geometric fluxes.

Acknowledgments: We are grateful to David Andriot, Andreas Deser, Oscar Loaiza-Brito, Dieter Lüst, Eran Palti, Erik Plauschinn, Felix Rennecke and Christian Schmid for discussion and Adolfo Guarino and the referee for useful comments about an earlier version of this paper. XG is supported by the MPG-CAS Joint Doctoral Promotion Programme. PS is supported by a postdoctoral research fellowship from the Alexander von Humboldt Foundation. 


\section{A The detailed form of the superpotentials}

In this appendix we present the explicit form of the superpotential when written out in terms of the individual fluxes and moduli.

\section{The type IIA superpotential}

Using

$$
\begin{aligned}
\overline{\mathfrak{H}}_{i j k}^{C}=\bar{H}_{i j k}+3 \bar{F}_{[\underline{i j}}^{m}\left(-i J_{c}\right)_{m \underline{k}]} & +3 \bar{Q}_{[\underline{i}}^{m n}\left(-i J_{c}\right)_{m \underline{j}}\left(-i J_{c}\right)_{n \underline{k}]} \\
& +\bar{R}^{m n p}\left(-i J_{c}\right)_{m[\underline{i}}\left(-i J_{c}\right)_{n \underline{j}}\left(-i J_{c}\right)_{p \underline{k}]},
\end{aligned}
$$

the NS-NS sector type IIA superpotential written out in terms of the fluxes and moduli reads (similar explicit expressions have been derived in [63])

$$
\begin{aligned}
& W_{\mathrm{NS}}=-\frac{i}{4} \int_{X} \overline{\mathfrak{H}}^{C} \wedge \Omega^{c} \\
& \frac{1}{4}\left[S \left(i \bar{H}_{135}-\bar{F}^{6}{ }_{13} T_{3}-\bar{F}^{2}{ }_{35} T_{1}-\bar{F}^{4}{ }_{51} T_{2}\right.\right. \\
&\left.\quad-i \bar{Q}_{1}{ }^{46} T_{2} T_{3}-i \bar{Q}_{3}^{62} T_{1} T_{3}-i \bar{Q}_{5}{ }^{24} T_{1} T_{2}+\bar{R}^{246} T_{1} T_{2} T_{3}\right) \\
&-U_{1}\left(i \bar{H}_{146}+\bar{F}^{5}{ }_{14} T_{3}-\bar{F}^{2}{ }_{46} T_{1}+\bar{F}^{3}{ }_{61} T_{2}\right. \\
&\left.\quad-i \bar{Q}_{1}{ }^{35} T_{2} T_{3}+i \bar{Q}_{4}{ }^{52} T_{1} T_{3}+i \bar{Q}_{6}{ }^{23} T_{1} T_{2}+\bar{R}^{235} T_{1} T_{2} T_{3}\right) \\
&-U_{2}\left(i \bar{H}_{236}+\bar{F}_{23}^{5} T_{3}+\bar{F}^{1}{ }_{36} T_{1}-\bar{F}^{4}{ }_{62} T_{2}\right. \\
&\left.\quad+i \bar{Q}_{2}{ }^{45} T_{2} T_{3}-i \bar{Q}_{3}{ }^{51} T_{1} T_{3}+i \bar{Q}_{6}{ }^{14} T_{1} T_{2}+\bar{R}^{145} T_{1} T_{2} T_{3}\right) \\
&-U_{3}\left(i \bar{H}_{245}-\bar{F}_{24}^{6} T_{3}+\bar{F}^{1}{ }_{45} T_{1}+\bar{F}^{3}{ }_{52} T_{2}\right. \\
&\left.\left.\quad+i \bar{Q}_{2}{ }^{36} T_{2} T_{3}+i \bar{Q}_{4}{ }^{61} T_{1} T_{3}-i \bar{Q}_{5}{ }^{13} T_{1} T_{2}+\bar{R}^{136} T_{1} T_{2} T_{3}\right)\right] .
\end{aligned}
$$

In the $\mathrm{R}-\mathrm{R}$ sector one finds

$$
\begin{aligned}
W_{\mathrm{R}}= & \frac{1}{4} \int_{X} e^{i J_{c}} \wedge \bar{G} \\
= & \frac{1}{4}\left[\bar{G}_{123456}^{(6)}+i \bar{G}_{3456}^{(4)} T_{1}+i \bar{G}_{1256}^{(4)} T_{2}+i \bar{G}_{1234}^{(4)} T_{3}\right. \\
& \left.\quad-\bar{G}_{12}^{(2)} T_{2} T_{3}-\bar{G}_{34}^{(2)} T_{1} T_{3}-\bar{G}_{56}^{(2)} T_{1} T_{2}-i T_{1} T_{2} T_{3} \bar{G}^{(0)}\right]
\end{aligned}
$$




\section{The type IIB superpotential}

In the type IIB example, the NS-NS part of the superpotential can be expanded as

$$
\begin{aligned}
W_{N S}= & -\frac{i}{4} \int_{X}\left(S \bar{H}+\bar{Q} \cdot J^{c}\right) \wedge \Omega_{3} \\
= & \frac{1}{4}\left[S \left(-i \bar{H}_{246}-\bar{H}_{146} U_{1}-\bar{H}_{236} U_{2}-\bar{H}_{245} U_{3}\right.\right. \\
& \left.+i \bar{H}_{136} U_{1} U_{2}+i \bar{H}_{235} U_{2} U_{3}+i \bar{H}_{145} U_{1} U_{3}+\bar{H}_{135} U_{1} U_{2} U_{3}\right) \\
+ & T_{1}\left(i \bar{Q}_{2}{ }^{35}+\bar{Q}_{1}{ }^{35} U_{1}-\bar{Q}_{2}{ }^{45} U_{2}-\bar{Q}_{2}{ }^{36} U_{3}\right. \\
& \left.+i \bar{Q}_{1}^{45} U_{1} U_{2}-i \bar{Q}_{2}{ }^{46} U_{2} U_{3}+i \bar{Q}_{1}{ }^{36} U_{1} U_{3}-\bar{Q}_{1}{ }^{46} U_{1} U_{2} U_{3}\right) \\
+ & T_{2}\left(i \bar{Q}_{4}{ }^{51}-\bar{Q}_{4}{ }^{52} U_{1}+\bar{Q}_{3}{ }^{51} U_{2}-\bar{Q}_{4}{ }^{61} U_{3}\right. \\
& \left.+i \bar{Q}_{3}{ }^{52} U_{1} U_{2}+i \bar{Q}_{3}{ }^{61} U_{2} U_{3}-i \bar{Q}_{4}{ }^{62} U_{1} U_{3}-\bar{Q}_{3}{ }^{62} U_{1} U_{2} U_{3}\right) \\
+ & T_{3}\left(i \bar{Q}_{6}{ }^{13}-\bar{Q}_{6}{ }^{23} U_{1}-\bar{Q}_{6}{ }^{14} U_{2}+\bar{Q}_{5}{ }^{13} U_{3}\right. \\
& \left.\left.-i \bar{Q}_{6}{ }^{24} U_{1} U_{2}+i \bar{Q}_{5}{ }^{14} U_{2} U_{3}+i \bar{Q}_{5}{ }^{23} U_{1} U_{3}-\bar{Q}_{5}{ }^{24} U_{1} U_{2} U_{3}\right)\right]
\end{aligned}
$$

and the R-R part as

$$
\begin{aligned}
W_{R}= & \frac{1}{4} \int_{X} \bar{G}^{(3)} \wedge \Omega_{3} \\
= & \frac{1}{4}\left[\bar{G}_{246}-i \bar{G}_{146} U_{1}-i \bar{G}_{236} U_{2}-i \bar{G}_{245} U_{3}\right. \\
& \left.-\bar{G}_{235} U_{2} U_{3}+\bar{G}_{145} U_{1} U_{3}+\bar{G}_{136} U_{1} U_{2}+i \bar{G}_{135} U_{1} U_{2} U_{3}\right]
\end{aligned}
$$

\section{B Details on the scalar potential}

The detailed computation of the scalar potential involves quite a number of individual terms. Recall that we compute an F-term scalar potential $V_{F}$ from the superpotential and a Kähler potential and compare the result with the dimensional reduction $V_{\text {kin }}=V_{\text {kin }}^{\mathrm{NS}}+V_{\text {kin }}^{\mathrm{R}}$ of the ten-dimensional kinetic terms.

For the interested reader, we provide some more details on the explicit computations, namely the number of individual terms in the scalar potential.

\section{Counting terms for type IIA}

For the type IIA setup, we consider four cases for which, in addition to all R-R fluxes, we have: 
(i) only $H_{i j k}$ flux turned-on

(ii) $H_{i j k}$ and geometric fluxes $F^{i}{ }_{j k}$ turned-on

(iii) $H_{i j k}, F^{i}{ }_{j k}$ and $Q_{k}{ }^{i j}$ fluxes turned-on

(iv) all fluxes turned-on

Then we find the number of terms listed in table 1

\begin{tabular}{|c|c|c|c|c|c|c|}
\hline $\begin{array}{c}\text { fluxes } \\
\text { turned-on }\end{array}$ & $V_{\mathrm{kin}}^{\mathrm{NS}}$ & $V_{\text {kin }}^{\mathrm{R}}$ & $V_{D}$ & $V_{\text {kin }}-V_{D}$ & $V_{F}$ & $\begin{array}{c}V_{F}-V_{\text {kin }}+V_{D} \\
\text { (to be removed by BIs) }\end{array}$ \\
\hline$H$ & 4 & 118 & 4 & 126 & 126 & 0 \\
\hline$H, F$ & 64 & 418 & 16 & 498 & 522 & $24: F^{2}=0$ \\
\hline$H, F, Q$ & 112 & 1138 & 28 & 1446 & 1686 & $\begin{array}{c}240 \\
48: F^{2}+H Q=0(12 \mathrm{BIs}) \\
48: Q^{2}=0(12 \mathrm{BIs}) \\
144: F Q=0(24 \mathrm{BIs})\end{array}$ \\
\hline$H, F, Q, R$ & 424 & 1630 & 32 & 2086 & 2422 & $\begin{array}{c}336 \\
96: F^{2}+H Q=0(12 \mathrm{BIs}) \\
48: Q^{2}+R F=0(12 \mathrm{BIs}) \\
192: F Q+H R=0(24 \mathrm{BIs})\end{array}$ \\
\hline
\end{tabular}

Table 1: Number of individual terms in the scalar potential for the type IIA orientifold.

Note that the case (i) is the standard example in type IIA with just the usual NS-NS and R-R fluxes turned on, whereas case (ii) reproduces the results of [22] with the inclusion of geometric flux. For all cases, after imposing the Bianchi identities, the kinetic terms reduce according to

$$
V_{\mathrm{kin}}=V_{\mathrm{kin}}^{\mathrm{NS}}+V_{\mathrm{kin}}^{\mathrm{R}}=V_{F}+V_{D}
$$

to the expected F-term potential plus a D-term, which combines with the D-term from the localized D6-branes and O6-planes. Using the R-R tadpole cancellation conditions, the entire D-term vanishes.

\section{Counting terms for type IIB}

For the type IIB example, we only need to distinguish two cases

(i) only $H_{i j k}$ flux turned-on 
(ii) $H_{i j k}$ and non-geometric fluxes $Q_{k}{ }^{i j}$ turned-on which are listed in table 2 .

\begin{tabular}{|c||c|c|c|c||c||c||c|}
\hline $\begin{array}{c}\text { fluxes } \\
\text { turned-on }\end{array}$ & $V_{\text {kin }}^{\mathrm{NS}}$ & $V_{\text {kin }}^{\mathrm{R}}$ & $V_{D 3}$ & $V_{D 7}$ & $V_{\text {kin }}-V_{D}$ & $V_{F}$ & $\begin{array}{c}V_{F}-V_{\text {kin }}+V_{D} \\
\text { (to be removed by BIs })\end{array}$ \\
\hline \hline$H$ & 76 & 277 & 8 & 0 & 361 & 361 & 0 \\
& 424 & 1630 & 8 & 24 & 2086 & 2422 & $\begin{array}{c}336 \\
168: H Q(24 \mathrm{BIs}) \\
168: Q^{2}=0(24 \mathrm{BIs})\end{array}$ \\
\hline
\end{tabular}

Table 2: Number of individual terms in the scalar potential for the type IIB orientifold.

It is interesting to observe that, in the most generic case when one includes all possible fluxes, the total number of terms in the type IIA and type IIB case exactly match in each column, including also the number of terms which get nullified via the Bianchi identities. This observation supports one of the main initial motivations for introducing non-geometric fluxes [6], namely the consistency of fluxes with T-duality. 


\section{References}

[1] S. Kachru, M. B. Schulz, P. K. Tripathy, and S. P. Trivedi, "New supersymmetric string compactifications," JHEP 0303 (2003) 061, hep-th/0211182.

[2] S. Hellerman, J. McGreevy, and B. Williams, "Geometric constructions of nongeometric string theories," JHEP 0401 (2004) 024, hep-th/0208174.

[3] A. Dabholkar and C. Hull, "Duality twists, orbifolds, and fluxes," JHEP 0309 (2003) 054, hep-th/0210209.

[4] C. Hull, "A Geometry for non-geometric string backgrounds," JHEP 0510 (2005) 065, hep-th/0406102.

[5] C. M. Hull, "Doubled Geometry and T-Folds," JHEP 0707 (2007) 080, hep-th/0605149.

[6] J. Shelton, W. Taylor, and B. Wecht, "Nongeometric Flux

Compactifications," JHEP 10 (2005) 085, hep-th/0508133.

[7] P. G. Camara, A. Font, and L. Ibanez, "Fluxes, moduli fixing and MSSM-like vacua in a simple IIA orientifold," JHEP 0509 (2005) 013, hep-th/0506066.

[8] G. Aldazabal, P. G. Camara, A. Font, and L. Ibanez, "More dual fluxes and moduli fixing," JHEP 0605 (2006) 070, hep-th/0602089.

[9] B. de Carlos, A. Guarino, and J. M. Moreno, "Complete classification of Minkowski vacua in generalised flux models," JHEP 1002 (2010) 076, 0911.2876 .

[10] U. Danielsson and G. Dibitetto, "On the distribution of stable de Sitter vacua," JHEP 1303 (2013) 018, 1212.4984.

[11] J. Blaback, U. Danielsson, and G. Dibitetto, "Fully stable dS vacua from generalised fluxes," 1301.7073.

[12] C. Damian, O. Loaiza-Brito, L. Rey, and M. Sabido, "Slow-Roll Inflation in Non-geometric Flux Compactification," 1302.0529.

[13] C. Damian and O. Loaiza-Brito, "More stable dS vacua from S-dual non-geometric fluxes," 1304.0792.

[14] J.-P. Derendinger, C. Kounnas, P. M. Petropoulos, and F. Zwirner, "Superpotentials in IIA compactifications with general fluxes," Nucl.Phys. B715 (2005) 211-233, hep-th/0411276. 
[15] J.-P. Derendinger, C. Kounnas, P. Petropoulos, and F. Zwirner, "Fluxes and gaugings: N=1 effective superpotentials," Fortsch.Phys. 53 (2005) 926-935, hep-th/0503229.

[16] G. Dall'Agata, G. Villadoro, and F. Zwirner, "Type-IIA flux compactifications and N=4 gauged supergravities," JHEP 0908 (2009) $018,0906.0370$.

[17] G. Aldazabal, D. Marques, C. Nunez, and J. A. Rosabal, "On Type IIB moduli stabilization and $\mathrm{N}=4,8$ supergravities," Nucl.Phys. B849 (2011) $80-111,1101.5954$.

[18] G. Aldazabal, W. Baron, D. Marques, and C. Nunez, "The effective action of Double Field Theory," JHEP 1111 (2011) 052, 1109.0290.

[19] D. Geissbuhler, "Double Field Theory and N=4 Gauged Supergravity," JHEP 1111 (2011) 116, 1109.4280.

[20] M. Graña and D. Marques, "Gauged Double Field Theory," JHEP 1204 (2012) 020, 1201.2924.

[21] G. Dibitetto, J. Fernandez-Melgarejo, D. Marques, and D. Roest, "Duality orbits of non-geometric fluxes," Fortsch.Phys. 60 (2012) 1123-1149, 1203.6562

[22] G. Villadoro and F. Zwirner, "N=1 effective potential from dual type-IIA D6/O6 orientifolds with general fluxes," JHEP 0506 (2005) 047, hep-th/0503169.

[23] G. Aldazabal, P. G. Camara, and J. Rosabal, "Flux algebra, Bianchi identities and Freed-Witten anomalies in F-theory compactifications," Nucl.Phys. B814 (2009) 21-52,0811.2900.

[24] G. Aldazabal, E. Andres, P. G. Camara, and M. Grana, "U-dual fluxes and Generalized Geometry," JHEP 1011 (2010) 083, 1007.5509.

[25] G. Dibitetto, A. Guarino, and D. Roest, "Charting the landscape of $\mathrm{N}=4$ flux compactifications," JHEP 1103 (2011) 137, 1102.0239.

[26] N. Hitchin, "Generalized Calabi-Yau manifolds," Quart.J.Math.Oxford Ser. 54 (2003) 281-308, math/0209099.

[27] M. Gualtieri, "Generalized complex geometry," math/0401221.

[28] M. Graña, R. Minasian, M. Petrini, and D. Waldram, "T-duality, Generalized Geometry and Non-Geometric Backgrounds," JHEP 0904 (2009) 075, 0807.4527. 
[29] A. Coimbra, C. Strickland-Constable, and D. Waldram, "Supergravity as Generalised Geometry I: Type II Theories," JHEP 1111 (2011) 091, 1107.1733 .

[30] C. Hull and B. Zwiebach, "Double Field Theory," JHEP 0909 (2009) 099, 0904.4664. 51 pages.

[31] C. Hull and B. Zwiebach, "The Gauge algebra of double field theory and Courant brackets," JHEP 0909 (2009) 090, 0908.1792.

[32] O. Hohm, C. Hull, and B. Zwiebach, "Background independent action for double field theory," JHEP 1007 (2010) 016, 1003.5027.

[33] O. Hohm, C. Hull, and B. Zwiebach, "Generalized metric formulation of double field theory," JHEP 1008 (2010) 008, 1006.4823.

[34] C. Hull, "Generalised Geometry for M-Theory," JHEP 0707 (2007) 079, hep-th/0701203.

[35] P. P. Pacheco and D. Waldram, "M-theory, exceptional generalised geometry and superpotentials," JHEP 0809 (2008) 123, 0804.1362.

[36] D. S. Berman and M. J. Perry, "Generalized Geometry and M theory," JHEP 1106 (2011) 074, 1008.1763.

[37] D. Andriot, M. Larfors, D. Lüst, and P. Patalong, "A ten-dimensional action for non-geometric fluxes," JHEP 1109 (2011) 134, 1106.4015.

[38] D. Andriot, O. Hohm, M. Larfors, D. Lüst, and P. Patalong, "A geometric action for non-geometric fluxes," Phys.Rev.Lett. 108 (2012) 261602, 1202.3060 .

[39] D. Andriot, O. Hohm, M. Larfors, D. Lüst, and P. Patalong, "Non-Geometric Fluxes in Supergravity and Double Field Theory," Fortsch.Phys. 60 (2012) 1150-1186, 1204.1979.

[40] R. Blumenhagen, A. Deser, E. Plauschinn, and F. Rennecke, "A bi-invariant Einstein-Hilbert action for the non-geometric string," Phys.Lett. B720 (2013) 215-218, 1210.1591.

[41] R. Blumenhagen, A. Deser, E. Plauschinn, and F. Rennecke, "Non-geometric strings, symplectic gravity and differential geometry of Lie algebroids," JHEP 1302 (2013) 122, 1211.0030.

[42] R. Blumenhagen, A. Deser, E. Plauschinn, F. Rennecke, and C. Schmid, "The Intriguing Structure of Non-geometric Frames in String Theory," 1304.2784. 
[43] G. Aldazabal, D. Marques, and C. Nunez, "Double Field Theory: A Pedagogical Review," 1305.1907.

[44] D. S. Berman and D. C. Thompson, "Duality Symmetric String and M-Theory," 1306.2643.

[45] R. Blumenhagen, A. Deser, E. Plauschinn, and F. Rennecke, "Bianchi Identities for Non-Geometric Fluxes - From Quasi-Poisson Structures to Courant Algebroids," Fortsch.Phys. 60 (2012) 1217-1228, 1205.1522.

[46] D. Geissbuhler, D. Marques, C. Nunez, and V. Penas, "Exploring Double Field Theory," 1304.1472.

[47] G. Dall'Agata and N. Prezas, "Scherk-Schwarz reduction of M-theory on G2-manifolds with fluxes," JHEP 0510 (2005) 103, hep-th/0509052.

[48] D. S. Berman, E. T. Musaev, D. C. Thompson, and D. C. Thompson, "Duality Invariant M-theory: Gauged supergravities and Scherk-Schwarz reductions," JHEP 1210 (2012) 174, 1208.0020.

[49] D. S. Berman and K. Lee, "Supersymmetry for Gauged Double Field Theory and Generalised Scherk-Schwarz Reductions," 1305.2747.

[50] T. R. Taylor and C. Vafa, "R R flux on Calabi-Yau and partial supersymmetry breaking," Phys.Lett. B474 (2000) 130-137, hep-th/9912152.

[51] T. W. Grimm and J. Louis, "The Effective action of type IIA Calabi-Yau orientifolds," Nucl.Phys. B718 (2005) 153-202, hep-th/0412277.

[52] I. Benmachiche and T. W. Grimm, "Generalized N=1 orientifold compactifications and the Hitchin functionals," Nucl.Phys. B748 (2006) 200-252, hep-th/0602241.

[53] O. Hohm and S. K. Kwak, "Frame-like Geometry of Double Field Theory," J.Phys. A44 (2011) 085404, 1011.4101.

[54] W. Siegel, "Two vierbein formalism for string inspired axionic gravity," Phys.Rev. D47 (1993) 5453-5459, hep-th/9302036.

[55] W. Siegel, "Superspace duality in low-energy superstrings," Phys.Rev. D48 (1993) 2826-2837, hep-th/9305073.

[56] N. Halmagyi, "Non-geometric Backgrounds and the First Order String Sigma Model," 0906.2891.

[57] D. Roytenberg, "Courant algebroids, derived brackets and even symplectic supermanifolds," math/9910078. 
[58] A. Font, A. Guarino, and J. M. Moreno, "Algebras and non-geometric flux vacua," JHEP 0812 (2008) 050, 0809.3748.

[59] O. Hohm, S. K. Kwak, and B. Zwiebach, "Unification of Type II Strings and T-duality," Phys.Rev.Lett. 107 (2011) 171603, 1106.5452.

[60] O. Hohm, S. K. Kwak, and B. Zwiebach, "Double Field Theory of Type II Strings," JHEP 1109 (2011) 013, 1107.0008.

[61] O. Hohm and S. K. Kwak, "Massive Type II in Double Field Theory," JHEP 1111 (2011) 086, 1108.4937.

[62] I. Jeon, K. Lee, and J.-H. Park, "Ramond-Ramond Cohomology and O(D,D) T-duality," JHEP 1209 (2012) 079, 1206.3478.

[63] F. Hassler and D. Lüst, "Non-commutative/non-associative IIA (IIB) Qand R-branes and their intersections," 1303.1413. 